\title{
Cerebral Magnetic Resonance Imaging Feasibility in Patients with Implanted Neurostimulation System for Deep Brain Stimulation
}

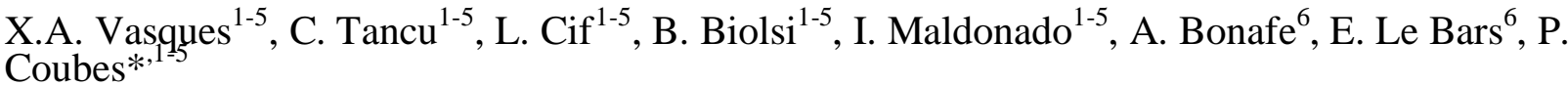

${ }^{1}$ CHRU Montpellier, Service de Neurochirurgie, Montpellier, France; ${ }^{2} I G F$, Montpellier, France; ${ }^{3}$ CNRS UMR5203, Montpellier, France; ${ }^{4}$ INSERM, U661, Montpellier, France; ${ }^{5}$ Université Montpellier I, Montpellier, France; ${ }^{6} \mathrm{CHRU}$ Montpellier, Service de Neuroradiologie, Montpellier France

\begin{abstract}
The goal of this study was to discuss the guidelines that permit patients with implanted deep brain stimulation (DBS) system to undergo Magnetic Resonance imaging (MRI) safely. We reported our experience of 226 MRI performed on 161 patients (134 dystono-dyskinetic syndromes and 27 Parkinson's disease) of which 32 reimplantations.

The stereotactic protocol used for the anatomical target localization in our center involves a pre- and postoperative MRI under general anaesthesia. The postoperative MRI allows measuring the possible error, checking the electrode position and the absence of surgical complication. No complications, no haemorrhages, no electrode displacement or fracture, no images similar to pallidotomy, and no neurological deficit were found related to MRI. We recorded only two minor hardware related complications occurring after MRI: (1) a stimulator damage after spinal MRI and (2) a parameter reset after cerebral MRI.
\end{abstract}

Clinical data confirm MRI feasibility in patients with implanted deep brain stimulation system.

\section{INTRODUCTION}

The use of implantable deep brain stimulation (DBS) system has become increasingly common in the treatment of refractory movement disorders including Parkinson's disease (PD) [1] and dystono-dyskinetic syndromes (DDS) [2-7]. In addition, several studies have examined the use of DBS in treating other neurological and psychiatric disorders including epilepsy [8], multiple sclerosis [9], depression [10], obsessive compulsive disorder [11] and Tourette syndrome [12].

The implantation of DBS electrodes is facilitated by using Magnetic Resonance imaging (MRI) guided stereotactic localization in order to achieve the most accurate location [13-16].

The stereotactic protocol developed in our center for the location of the anatomical target, notably the sensory-motor segment of the globus pallidus internus (GPi), includes a preand postoperative MRI with a Leksell Frame under general anaesthesia. The use of MRI enables to visualize the GPi borders directly and to select the target inside the limits of the GPi, taking into account individual anatomical variations. Notably due to the young age, poor general state of health, and chronic hyperkinetic condition of children with severe DDS, it was necessary to develop a short procedure that could be performed with the child in a state of general anaesthesia and during which target localization was based only on 3D MR imaging, without concomitant intraoperative microelectrode recordings. This procedure proved to be at low risk of complications ( $0 \%$ bleeding) and high precision

*Address correspondence to this author at the Unité de Recherche sur les Mouvements Anormaux, Hôpital Gui de Chauliac, Service de Neurochirurgie, 80 Avenue Augustin Fliche, 34295 Montpellier cedex 05, France; Tel: +334673374 64; Fax: +3346733 74 64;

E-mail: p-coubes@chu-montpellier.fr
[15-17]. The postoperative MRI allows checking the electrode position and detecting any error caused by MR imaging distortions.

In November 2005, the Medtronic manufacturer changed the safety parameters for the cerebral MRI in patients with a DBS system. Medtronic published new parameters after an incident occurred in the United States. For a given sequence of MRI, a high Specific Absorption Rate (SAR) could induce, according to the company, an immoderate and dangerous heating of the electrodes and/or the implanted stimulation system. Medtronic has adopted new limits for safe exposure to MRI devices: 0.1 Watt per kilogram $(\mathrm{W} / \mathrm{Kg})$ instead of $0.4 \mathrm{~W} / \mathrm{Kg}$.

In our practice, the MRI feasibility on patients with implanted DBS system was never reconsidered. It is necessary to differentiate (1) the MRI to check the accuracy of the electrodes (neurostimulators are not implanted, the distal extremity of the electrodes was isolated) from (2) the diagnostic MRI (all the system is implanted). We report our experience of 226 MRI performed on 161 patients of which 32 reimplantations (134 DDS and 27 PD). The implanted DBS system includes the electrode (4 contacts numbered from 0 to 3 , radius: 0.635 , contact height: $1.5 \mathrm{~mm}$, space between contacts: $0.5 \mathrm{~mm}$; ref: 3389 , Medtronic) and the internal pulse generator (IPG, Model 7424, 7425, 7426, Medtronic).

We consider that it is important to report our experience in order to demonstrate the innocuousness of the direct targeting using MRI.

\section{MATERIALS AND METHODOLOGY}

\section{Patient Population}

One hundred thirty four patients (mean age $24 \pm 12.7$ years, youngest 5 years old, oldest 74 years old) presenting dystonia and/or dyskinesia were treated by bilateral chronic 
electrical stimulation of GPi. The 134 DDS can be classified as: 68 primary DDS, 32 secondary DDS, 22 heredodegenerative and 12 dystonia plus.

One hundred sixty four surgical procedures were performed for dystono-dyskinetic patients (134 first surgery + 30 reimplantations) with 321 leads implanted within the GPi. Among the 134 patients, two had one electrode implanted, 120 were bilaterally implanted and 12 had four electrodes implanted.

Twenty seven patients (mean age $61 \pm 10.2$ years) with Parkinson's disease were implanted. The total number of surgical procedures in these patients was 29 (27 first surgery and 2 reimplantations) with 57 electrodes implanted. All the parkinsonian patients were bilaterally implanted. For PD, the target was the subthalamic nucleus (STN) in 15 procedures and the postero-ventral GPi in 14 procedures.

Three hundred seventy eight electrodes were implanted during 193 procedures (161 first procedures and 32 reimplantations). Two hundred twenty six MRI were performed for the postoperative checking. Among these 226 MRI, 163 (161 first surgery, 1 preoperative MRI for reimplantation, and 1 postoperative MRI for reimplantation) were performed with the leads implanted and without neurostimulators, 63 (31 preoperative MRI for reimplantation, 29 postoperative MRI for reimplantation, and 3 MRI outside the neurosurgical protocol) were performed with leads and neurostimulators implanted.

Thirty two surgeries for reimplantation were conducted after the first implantation in 23 patients. The reasons for supplementary electrodes implantation can be divided either in complications (19 infectious or hardware related complications) or necessary adaptation of DBS to chronic disease (13 electrode additions).

\section{Statistical Analysis}

The descriptive analysis was performed using the Mean \pm Standard Deviation for quantitative variables. Discrete variables are summarized as absolute numbers and percentages. For precise assessment of therapeutic efficacy, each patient is evaluated, pre- and postoperatively, applying the two sections (motor and disability scores) of the Burke-MarsdenFahn Dystonia Rating Scale (BMFDRS) at given intervals for DDS [18]. The Unified Parkinson's Disease Rating Scale (UPDRS) was used for parkinsonian patients. The percentage of improvement is calculated based on the maximal possible gain [(Preoperative score - Postoperative score)/ Preoperative score $]^{*} 100$. In order to study the significance of the difference between the pre- and postoperative scores, a bilateral Wilcoxon Signed Rank Tests was used. All the statistical analyses were performed using the R free software.

\section{Surgical Technique}

The MR imaging-based stereotactic procedure developed and validated in our center has been previously described elsewhere [13].

Considering the poor general condition and the permanent restless situation of most patients presenting DDS, especially children, we developed a stereotactic procedure under general anaesthesia. The procedure is based solely on 3D-MR imaging for target localization without intra- operative microelectrode recordings or clinical control allowing the procedure to be shortened to 1 hour per electrode.

General anesthesia is induced before placing the MRcompatible stereotactic frame (Leksell G frame; Elekta Instruments).

Following the visual recognition of the target boundaries on preoperative MRI, the neurosurgeon selects the target using a 3D-cursor. The electrode implantation is achieved under real-time strict-profile radioscopic control to prevent any modification of the electrode position until the end of the procedure.

The excess length of the electrode is wrapped around the skull burr hole. The number of loops is variable (0.5 to 3 ) with a mean value of 2 loops (Fig. 1) per electrode (diameter: $2.5 \mathrm{~cm}$ ). Immediately after electrode implantation, a control MRI is performed, with the stereotactic frame in place. A postoperative MRI is performed in order to record the electrode's position, document any error caused by MRI distortions and detect any hemorrhagic complication.

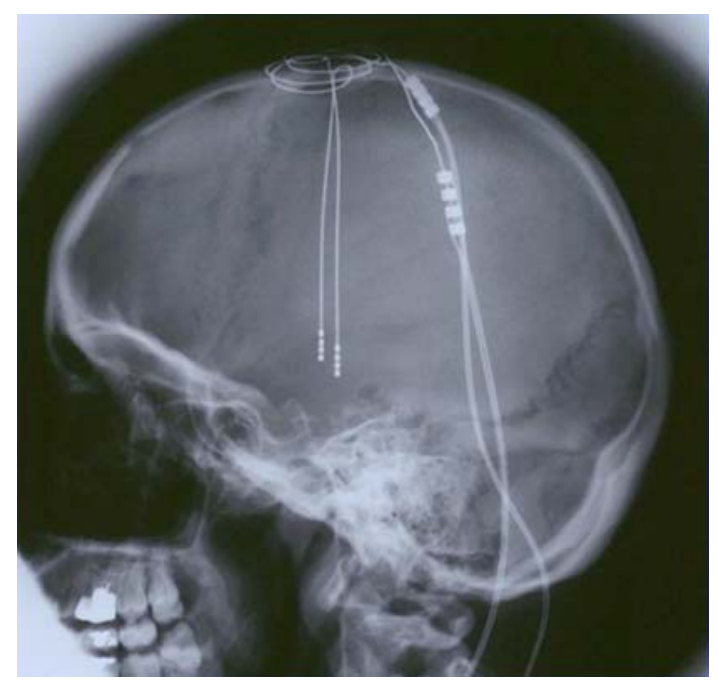

Fig. (1). Loops around the skull burr hole.

Five days after the implantation of the electrodes, a second surgery is performed for the IPG implantation in the abdominal area, also under general anesthesia. The excess length of the extensions is wrapped around the IPG with a mean value of 2 loops (Fig. 2) per IPG (diameter: $3.5 \mathrm{~cm}$ ).

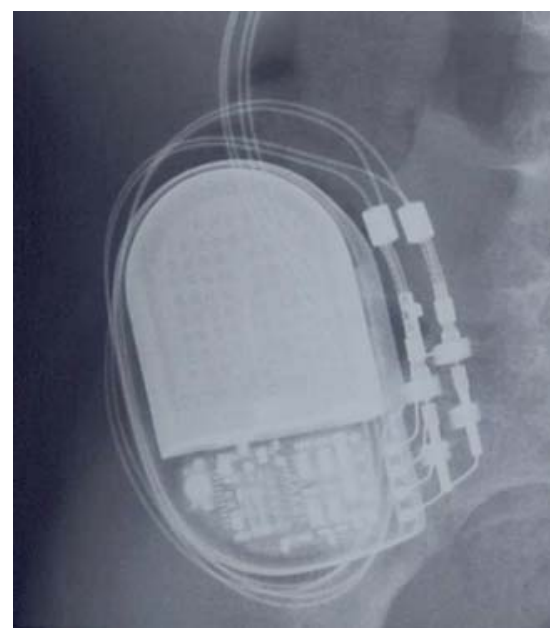

Fig. (2). Loops around the neurostimulator. 
Table 1. MRI Acquisition Protocol (Philips Intera 1.5T)

\begin{tabular}{|c|c|c|}
\hline Sequence & T1 & $\mathbf{T 2}$ \\
\hline \multicolumn{3}{|l|}{ Geometry } \\
\hline Coil Selection & Head & Head \\
\hline Homogeneity Correction & None & None \\
\hline Field Of View (mm) & 256 & 256 \\
\hline Foldover suppression & No & No \\
\hline Matrix Scan & 256 & 256 \\
\hline Reconstruction & 256 & 256 \\
\hline Stacks & 1 & 1 \\
\hline Slices & 126 & 30 \\
\hline Slice thickness (mm) & 1.5 & 1.5 \\
\hline Slice orientation & Transverse & Transverse \\
\hline Patient Position & $\begin{array}{l}\text { Supine: head } \\
\text { first }\end{array}$ & Supine: head first \\
\hline \multicolumn{3}{|l|}{ Contrast } \\
\hline Scan mode & $3 \mathrm{D}$ & $2 \mathrm{D}$ \\
\hline \multirow{2}{*}{ Pulse sequence } & Fast Field Echo & Spin Echo \\
\hline & Flip angle: $25^{\circ}$ & Flip angle: $90^{\circ}$ \\
\hline Fast Imaging Mode & None & Turbo Spin Echo \\
\hline Echoes & 1 & 1 \\
\hline Partial echo & Yes & No \\
\hline \multirow{2}{*}{ Scan timing (ms) } & TE: 5.5 & TE: 110 \\
\hline & TR: 25 & TR: 4200 \\
\hline \multicolumn{3}{|l|}{$\underline{\text { Motion }}$} \\
\hline Cardiac synchronization & no & no \\
\hline Respiratory compensation & no & no \\
\hline Flow compensation & yes & no \\
\hline NSA (Nex) & 1 & 4 \\
\hline \multicolumn{3}{|l|}{ Other information } \\
\hline SAR (W/Kg) / SAR level & $1.9 / 0$ & $1.6 / 0$ \\
\hline Total scan duration & $17: 10.5$ & 14:33.6 \\
\hline Measured voxel size $\mathrm{M} / \mathrm{P} / \mathrm{S}$ (mm) & $1 / 1 / 1.5$ & $1 / 1.02 / 1.5$ \\
\hline $\begin{array}{l}\text { Reconstructed voxel size M/P/S } \\
(\mathrm{mm})\end{array}$ & $1 / 1 / 1.5$ & $1 / 1 / 1.5$ \\
\hline SAR mode & High & High \\
\hline B1 mode & Default & Default \\
\hline Gradient mode & Default & Default \\
\hline SofTone mode & No & No \\
\hline
\end{tabular}

\section{MRI Acquisition}

Since August 2002, MRI acquisitions have been performed on a 1.5-T Philips Gyroscan Intera system adapted for receiving the stereotactic frame. The system is equipped with a transmit radiofrequency (RF) body coil and a receive radiofrequency head coil. A T1 3D Fast Field Echo (FFE) sequence is performed with the whole body SAR specified by the manufacturer Philips of $1.9 \mathrm{~W} / \mathrm{Kg}$, and a T2 Turbo Spin Echo (TSE) sequence with a SAR of $1.6 \mathrm{~W} / \mathrm{Kg}$ for both the pre- and postoperative examinations (Table 1). These two sequences were implemented without injection of gadolinium.

Up to 2002, we used a 1.5-T SIGNA General Electric. (General Electric, Paris, France) system with a transmit/receive RF head coil. A 3D-T1 Spoiled Gradient-recalled echo (SPGR) sequence was used (Table 2).

Table 2. MRI Acquisition Protocol (General Electric 1.5 T)

\begin{tabular}{|c|c|}
\hline Patient position & Head first \\
\hline Patient orientation & Supine \\
\hline Landmark & On the frame \\
\hline Coil selection & Head \\
\hline Scan plane & Axial \\
\hline Image mode & $3 \mathrm{D}$ \\
\hline \multirow{3}{*}{ Pulse sequence } & Spoiled Gradient-recalled echo \\
\hline & Variable \\
\hline & Bandwith \\
\hline \multirow{3}{*}{ Imaging options } & Extended dynamic range \\
\hline & Graphic RX \\
\hline & Flip angle: $25^{\circ}$ \\
\hline \multirow{4}{*}{ Scan timing } & $\mathrm{TE}=9.0 \mathrm{~ms}$ \\
\hline & $\mathrm{TR}=29.0 \mathrm{~ms}$ \\
\hline & Pre-scan options \\
\hline & Auto-shim \\
\hline \multirow{3}{*}{ Scan setup } & Auto CF \\
\hline & Water \\
\hline & Receive bandwith: $15.63 \mathrm{kHz}$ \\
\hline \multirow{2}{*}{ Scanning range } & Slice thickness: $1.5 \mathrm{~mm}$ \\
\hline & Slices: 124 \\
\hline \multirow{2}{*}{ Acquisition matrix: } & (frequency): 256 \\
\hline & (phase): 256 \\
\hline Frequency direction & $\mathrm{A} / \mathrm{P}$ \\
\hline \multirow{2}{*}{ Phase FOV } & 1 \\
\hline & $260 \mathrm{~mm}$ \\
\hline Imaging time & $1 \mathrm{Nex} 16.43$ \\
\hline Contrast & No \\
\hline
\end{tabular}

The MR images were acquired with the head frame in place. The reconstruction program processed images ac- 
Table 3. Neurostimulators Settings for MRI

\begin{tabular}{|c|c|}
\hline Parameters & Settings \\
\hline \hline Stimulation output & OFF (all programs) \\
\hline Stimulation mode & Bipolar (all programs) \\
\hline Amplitude & 0 Volts (all programs) \\
\hline Magnetic (reed) switch & Disabled (Kinetra model 7428 only) \\
\hline Other parameters & At the minimal level \\
\hline
\end{tabular}

quired on a $256 \times 256$ square matrix with a $256 \mathrm{~mm}$ wide field of view (FOV).

Control studies used to assess the homogeneity of the main magnetic field and the calibration of the gradients were performed the day before the stereotactic operation. The distortions were controlled at the periphery of the FOV using the G-frame localizer as a phantom and at the center of the FOV using the anterior and posterior commissures as landmarks [15]. All these control studies were in agreement with

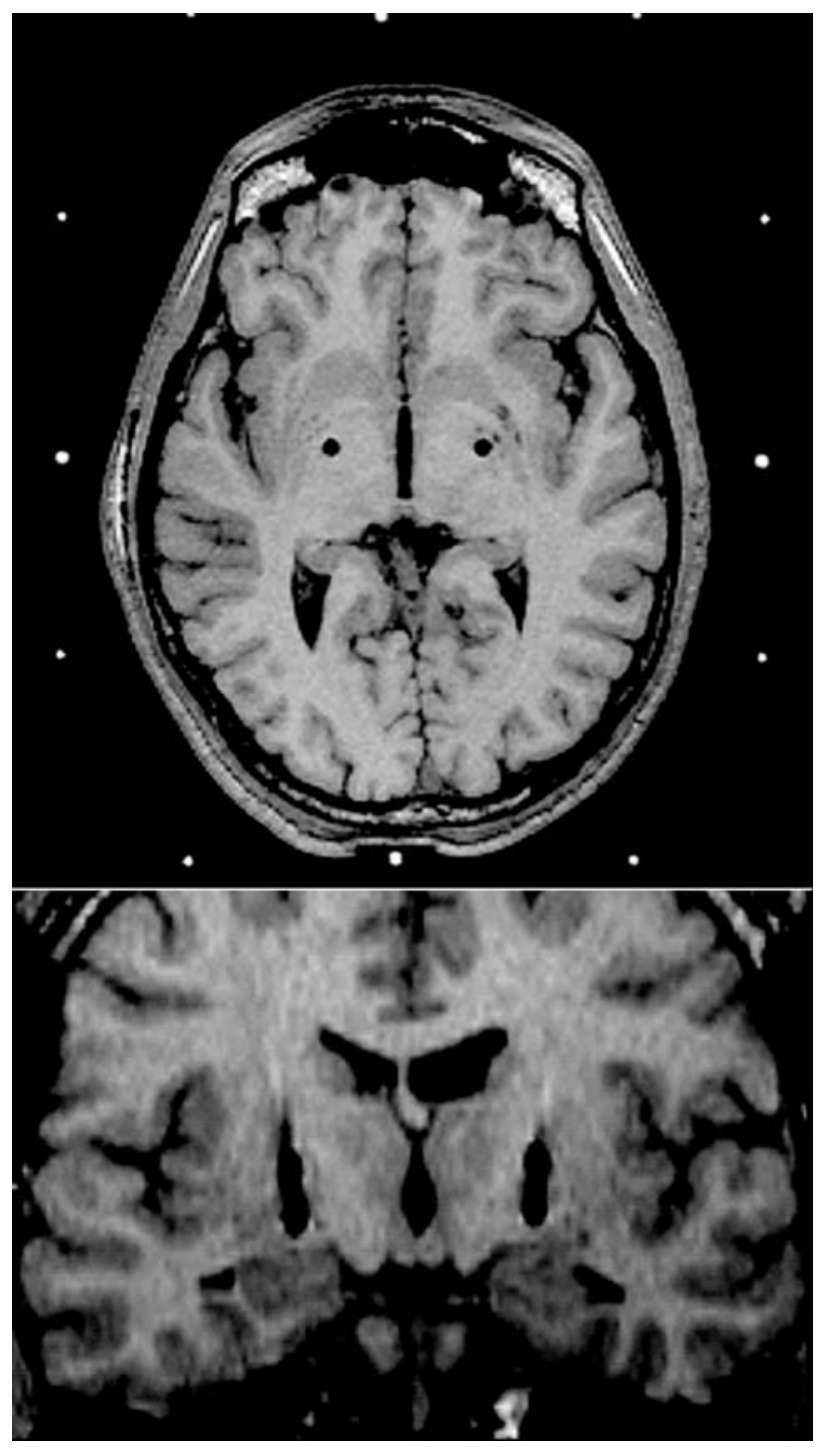

Fig. (3). Electrode artifact visualized as a hypo signal on T1 sequence (axial and coronal). the European patterns. The procedure included a control with a quality-assessment phantom.

\section{MRI Performed on Patients with Implanted Deep Brain Stimulation System}

One hundred fifty two $(67.25 \%)$ MRI were performed with Philips Gyroscan Intera MR system, and 74 (32.75 \%) with SIGNA General Electric MR system. We distinguish: MRI performed with electrodes not connected to the neurostimulators $(163,72.1 \%)$, and those with all the system implanted after reimplantation or diagnostic MRI outside the implantation protocol $(63,27.9 \%)$. Before performing the MRI, the IPG are switched off and the settings are programmed as is indicated in Table $\mathbf{3}$. These precautions were taken at the beginning of our activity, for elementary care, because of the knowledge of the metal exposition under magnetic field.

\section{Postoperative MRI Analysis}

All MRI were analysed by at least two physicians: a neurosurgeon and a radiologist. The postoperative MRI was performed using the same imaging protocol as that used for the preoperative MRI. Using the postoperative MRI, the electrodes position, the possible errors due to distortions and hemorrhagic complications were checked. Analysis was performed for screening thermical lesions around the electrodes.

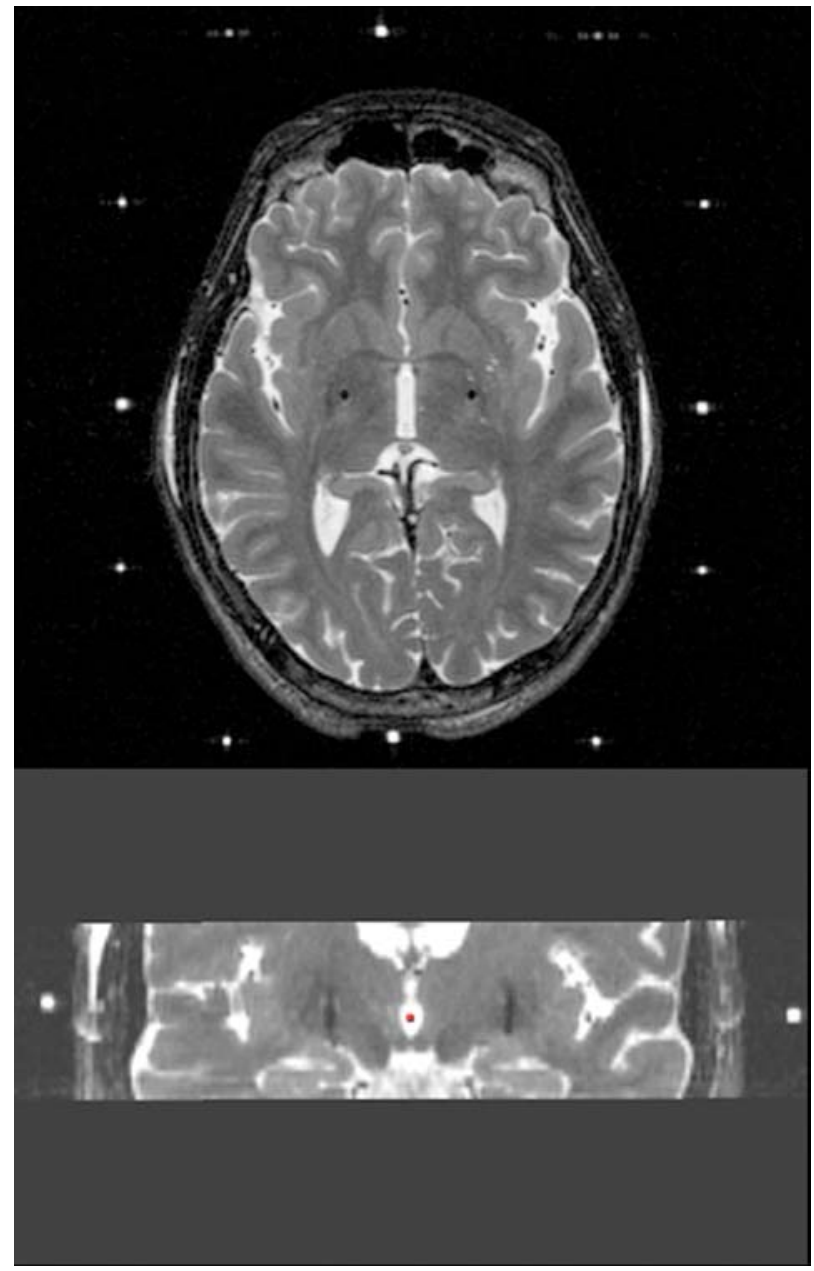

Fig. (4). Electrode artifact visualized as a hypo signal on T2 sequence (axial and coronal). 
On T1 (Fig. 3) and T2 (Fig. 4) sequences, the electrode was represented by an ellipsoidal artefact in hypo signal. The artefact dimensions were larger and longer than the real dimensions of the electrode. The dimensions of the artefact were smaller on $\mathrm{T} 2$ than on $\mathrm{T} 1$ sequence (Table 4). A potential thermical lesion produced by electrode heating would show a similar image to a pallidotomy. The lesions of pallidotomy performed with a thermical probe $\left(80^{\circ}\right)$ are visualized in hyper signal on T2 sequence and hypo signal on T1 sequence (Fig. 5).

Table 4. Electrode and Artifact Dimensions

\begin{tabular}{|c|c|c|}
\hline & Diameter & Total lenght \\
\hline \hline Electrode 3389 Medtronic & $1,27 \mathrm{~mm}$ & $7,5 \mathrm{~mm}$ \\
\hline T1 weighted artifact & $4,5 \mathrm{~mm}$ & $14-15 \mathrm{~mm}$ \\
\hline T2 weighted artifact & $2,5 \mathrm{~mm}$ & $12-13 \mathrm{~mm}$ \\
\hline
\end{tabular}

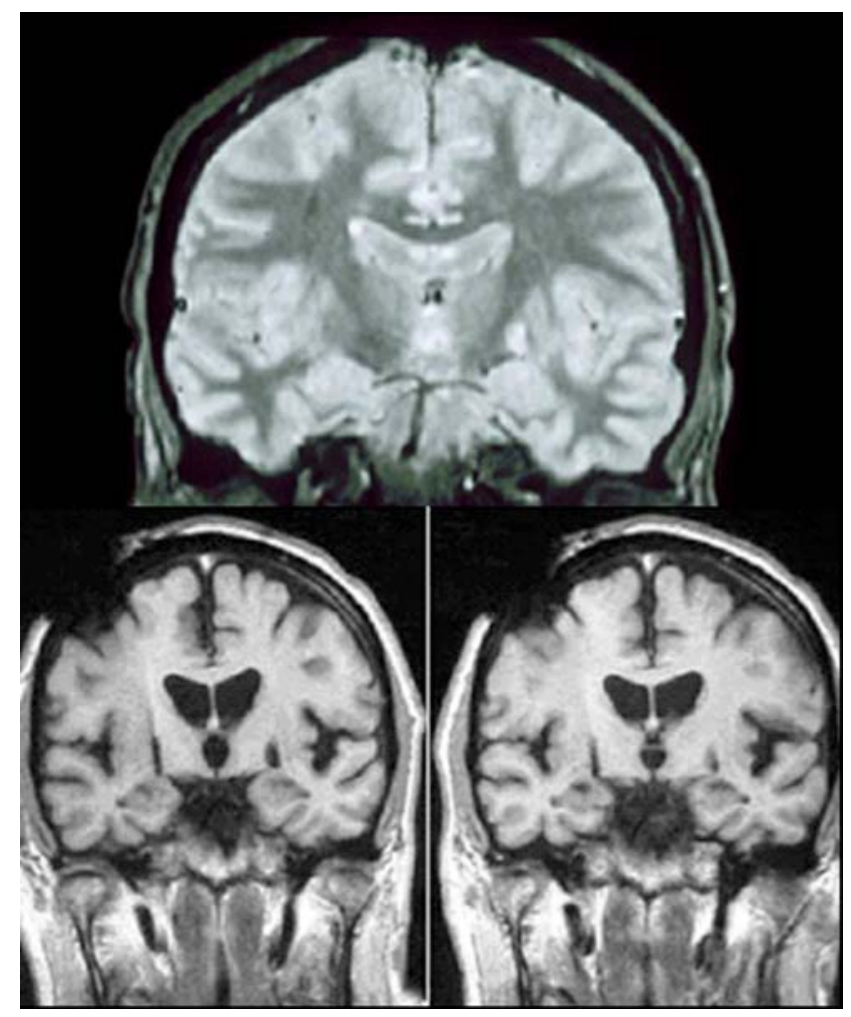

Fig. (5). Pallidotomy visualized as a hyper signal on T2 sequence (at the top). The electrode in the left side and the pallidotomy in the right side are visualized by a hypo signal on a $\mathrm{T} 1$ sequence (at the bottom).

\section{Intra- and Postoperative MRI Clinical Examination}

All the scans are supervised by four individuals (one neurosurgeon, one anaesthesiologist, one neuroradiologist and one MR technician constantly present). The postoperative clinical evaluation could reveal a neurological deficit caused by a potential thermical lesion. Absence of surgical complications was checked on the postoperative MRI. The absence of pallidotomy-like effect was also checked by comparing the preoperative clinical ratings with the postoperative clinical assessment previously to the switch-on of the neurostimulation devices. A lesion produced by electrode heat- ing would produce the same clinical effect as a therapeutic pallidotomy. Before choosing the electrical settings for chronic stimulation, a stimulation system checking, integrative part of the patient follow-up protocol was systematically performed after the postoperative MRI. Each contact of the electrode was tested separately. For the immediate postoperative assessment, patients were examined by three physicians (L.C., B.B. and P.C.) who arrived at a consensus opinion on the absence of neurological deficits (Table 5) due to the surgical procedure (including the postoperative MRI) and the absence of a pallidotomy-like effect. The examinations were performed daily during the postoperative hospital stay, monthly during the first year and at intervals of three months thereafter. The pre- and postoperative absolute scores of the BFMDRS were assigned.

Table 5. Immediate Postoperative Clinical Examination. Normal Clinical Findings for the 161 Patients. Only Two Minor Hardware Related Complications Occurring After MRI: A Stimulator Damage after a Spinal MRI and a Parameter Reset After Cerebral MRI

\begin{tabular}{|l|}
\hline Neurological examination \\
\hline Mental Status \\
\hline Level of Consciousness \\
\hline Orientation (time, person, place) \\
\hline Memory (3 items recall) \\
\hline Judgement (abstraction) \\
\hline Cognition \\
\hline Cranial nerves \\
\hline Language \\
\hline Motor \\
\hline Sensory \\
\hline Cerebellar \\
\hline Hardware related complications \\
\hline Parameter reset \\
\hline Hardware damage \\
\hline IPG reprogramming \\
\hline Displacement \\
\hline Warmth sensation over the material trajectory \\
\hline Erythema over the material trajectory \\
\hline
\end{tabular}

\section{RESULTS}

\section{Postoperative MRI Analysis}

After the analysis of all the postoperative MRI, no image compatible with a pallidotomy was found. No hyper intense $\mathrm{T} 2$ weighted images and no hypo intense $\mathrm{T} 1$ weighted images around the artefact of the electrode were found. No case of haemorrhages in our patients was found. No electrode displacements or fractures due to MRI were found.

63 MRI were performed with all the DBS system implanted and no images comparable to a thermal lesion were found. 


\section{Absence of any Supplementary Neurological Deficit}

We did not record any supplementary neurological deficit (transient or permanent) in our series of patients. Preoperatively, the patients with primary DDS evaluated by the BMFDRS were rated respectively at $53.7 \pm 17.3$ and $14.1 \pm 7.9$ for the motor and disability scores and, before the switch-on of the neurostimulators, the patients were rated respectively at $53.8 \pm 25.7$ and $14.1 \pm 7.9$. No significant differences were found between the preoperative scores and the scores before the switch-on of the neurostimulators (motor score: $p=0.171$ and disability score: $\mathrm{p}=0.609$ ).

Preoperatively, the other patients were rated respectively at $56.1 \pm 25.8$ and $15.8 \pm 8.1$ for the motor and disability scores and, before the switch-on of the neurostimulators, the patients were rated respectively at $55.3 \pm 25.6$ and $15.5 \pm 7.9$. No significant differences were found between the preoperative scores and the scores before the switch-on of the neurostimulators (motor score: $\mathrm{p}=0.453$ and disability score: $\mathrm{p}=0.177$ ).

The clinical improvement under DBS was statistically significant and increased with time. The patients with primary DDS evaluated by the BMFDRS at one-year follow-up improved respectively at $79 \pm 19 \% \quad(\mathrm{p}<0.0001)$ and $67.1 \pm 29.8 \%(\mathrm{p}<0.0001)$ for the motor (mean postoperative score at one-year follow-up: 11.26 \pm 14 ) and disability (mean postoperative score at one-year follow-up: 4.64 \pm 5.54 ) scores. For the other dystono-dyskinetic patients, the improvement was rated respectively at $42.7 \pm 31.4 \% \quad(\mathrm{p}<0.0001)$ and $32.5 \pm 29.8 \%(\mathrm{p}<0.0001)$ for the motor (mean postoperative score at one-year follow-up: $32.13 \pm 17.7)$ and disability (mean postoperative score at one-year follow-up: 10.7 \pm 5.7 ) scores. For the patients with PD, the mean global improvement at one-year follow-up evaluated by UPDRS III was of $62.3 \%(\mathrm{p}<0.001)$.

\section{Absence of Pallidotomy-Like Effect}

Previous to the electrical stimulation, the clinical improvement was not significant. For all patients, improvement (BMFDRS score) was not significant between electrode implantation and the beginning of the stimulation. 20 patients were evaluated by an OFF test (stimulation stopped). The loss of the clinical improvement evaluated by the difference between the relative BMFDRS score with stimulation ON and the score without stimulation was $30 \pm 55 \%$. A recurrence of the dystono-dyskinetic symptoms and a BMFDRS increase was observed in all patients in whom the stimulators had reached their end of life.

\section{Evaluation of Hardware Related Complications}

Only two hardware related complications were recorded in our population: (1) a stimulator (Itrel III) damage after a spinal MRI and (2) a parameter reset (Itrel III) after a cerebral MRI out of the surgical protocol. In these two cases, we did not observe any clinical consequence. Furthermore, the patients were conscious during acquisition and did not report pain or other sensations. Two other cases of spontaneous switch-off of the Itrel III stimulators were recorded without any known external influences. It seems that this type of parameters reset and/or spontaneous stop is frequent outside MRI performance.

\section{DISCUSSION}

The SAR is a measure of the rate at which radio frequency energy is absorbed by the body when exposed to radio-frequency electromagnetic field. Because of the tissue heterogeneity, direct SAR measurement is not possible. However, SAR measurement can be performed in homogeneous liquid and numerically evaluated in biological tissues. A wide range of factors can influence the SAR during MRI including RF, type of coil, concerned tissue volume, and body orientation in respect to vectors field. SAR calculation varies between different models of MR systems, algorithms of calculation, coil design and manufacturers [19]. Furthermore, the mathematical models and algorithms of calculation are not available or published because of industry property. The potential effects of MRI on DBS system are multiple. The RF field, the time-varying magnetic gradient field and the static magnetic field may induce a heating and a neurostimulator reprogramming or reset. It has not been possible, to date, to perform an accurate calculation of the heating during MRI.

\section{Electrode Heating During MRI}

The most important safety issue is the electrode heating during MRI. At the frequencies of interest in MRI, some of the RF energy is absorbed and converted to heat. During a MRI, the power deposited by RF pulses depends on a wide range of factors including the power and the duration of the $\mathrm{RF}$, the type of the RF transmitter coil used, the volume of tissue imaged, and the electrical resistivity of the tissues. During MRI on patients with a DBS system implanted, the magnetic field gradients and the radiofrequency field could be the origin of an induced voltage. This induced voltage produces a current which flows in a conductor involving a local heating. Several factors may influence the degree of heating such as the geometrical structure of the electrodes, the placement in the brain or the insulation of the electrodes.

A temperature elevation of more than $5^{\circ} \mathrm{C}$ causes reversible lesions of the neurons [20]. A temperature of more than $50^{\circ} \mathrm{C}$ causes irreversible lesions around the electrodes similar to a pallidotomy $\left(80^{\circ}\right.$ during one minute).

Several evaluations of MRI-related heating for neurostimulation systems used for DBS have been published [19, 21-26]. Significant temperature elevations were found in furthest conditions in contradiction with manufacturer's advice which cannot be applied in clinical practice.

Concentric loops placed around the burr hole seem to reduce MRI-related heating of electrodes [23]. Rezai et al. [25] showed that the temperature elevation was dependent on the type of radio frequency coil, level of SAR used and how the electrodes were positioned. The most important temperature elevation found in this study was of $25.3^{\circ} \mathrm{C}$ with the following configuration: (1) 4 loops around the neurostimulators; (2) 0 loops around the burr hole; (3) a SAR value of $3.9 \mathrm{~W} / \mathrm{Kg}$ with a transmit/receive body coil.

Finelli et al. [24] reported that MRI-related heating for clinical pulse sequences (including fast spin-echo, gradientecho and echo-planar imaging) using a transmit/receive RF head coil was correlated linearly with local SAR values for single and multislice fast spin-echo images. The authors indicate that the sequences performed at local SARs below 2.4 
W/kg (whole-body averaged SAR of $0.09 \mathrm{~W} / \mathrm{kg}$ ) should be safe from a MRI-related heating point of view. The temperature elevation is of approximately 0.9 times the local SAR value.

The neurostimulation systems were programmed to the boff Q mode (i.e., no stimulation was delivered) and set to 0 $\mathrm{V}$ according to the manufacturer's recommendation for patients with this device undergoing an MRI procedure (Medtronic).

Baker et al. [19] studied the temperature change at the electrode contacts using two different transmit/receive body coils on two different generation 1.5-Tesla MR systems from the same manufacturer. The result of the study revealed marked differences across two MR systems at the level of radiofrequency-induced temperature changes per unit of whole body SAR for a conductive implant.

These data suggest that the temperature elevations were clinically insignificant using clinical sequences for brain imaging. Consequently using SAR to guide MR safety recommendations for neurostimulation systems across different MR systems is not relevant. The calculation or estimation of the SAR values can vary on the basis of the model type and software of the MRI system. This is an issue of major interest because safety information identified to prevent heating for an implant, determined for a given MRI unit, may not be applied to another MRI unit, even if it is from the same manufacturer [19].

\section{Clinical Data Confirm MRI Feasability}

Spiegel et al. [27] reported that a 73-year-old patient with bilateral implanted DBS electrodes for Parkinson's disease showed dystonic and partially ballistic movements of the left leg immediately after undergoing a MRI of the head. This scan was performed with a transmit/receive head coil on a 1.0-T MRI system with the leads externalized and not connected to pulse generators. An important electrode heating and brain damage induced by MRI was published by Henderson et al. [28]. Henderson et al. [28] reported a severe neurological deficit (hemiplegia and aphasia) following electrodes heating during spinal MRI in a patient with a (DBS) system implanted for Parkinson's disease. The operation mode of the neurostimulation system during the MRI examination was unknown. Multiple scan sequences were performed with a 1.0-T MRI system and a transmit/receive body radiofrequency coil. In comparison to our protocol, we can note the use of a 1.0-T MRI instead of a 1.5-T MRI. The neurostimulators must be switch-off with 0 Volt during MRI which is not specified in this publication. Several studies $[23,25]$ demonstrated that temperature elevations were inversely proportional to the number of loops wrapped around the skull burr hole. Therefore, the implantation of neurostimulators in the upper thoracic area with an extension of 66 centimeters for adult patient (as it is the case in this article) doesn't allow these loops to be inserted.

Even if our study is limited by not assessing heating in a phantom, our experience with 226 MRI scans performed on 161 patients implanted with a deep brain stimulation system and the clinical results obtained during the last ten years confirm the innocuousness of the method used.

\section{CONCLUSION}

According to our surgical protocol and experience using it, we confirmed the validity of the method. Therefore, we can question the legitimacy of the unilateral decision by the industrial companies without preliminary evaluation of the medical impact and scientific consultation. In consequence, a study in collaboration with MRI manufacturers should be conducted in order to understand the ratio between the "applied SAR" and the "shown SAR" by the MRI and to adapt the protocols for each center. Our MRI protocol for patients with implanted DBS system joins up with other centers. Evaluations of MRI-related heating for neurostimulation systems used for DBS should be carried-out in each center in order to confirm the patient's safety.

\section{ACKNOWLEDGEMENTS}

We acknowledge Professor Marwan Hariz for the pallidotomy images contribution.

\section{REFERENCES}

[1] Benabid AL, Chabardes S, Seigneuret E. Deep-brain stimulation in Parkinson's disease: long-term efficacy and safety - What happened this year? Curr Opin Neurol 2005; 18(6): 623-30.

[2] Cif L, El Fertit H, Vayssiere N, et al. Treatment of dystonic syndromes by chronic electrical stimulation of the internal globus pallidus. J Neurosurg Sci 2003; 47(1): 52-5.

[3] Coubes P, Cif L, El Fertit H, et al. Electrical stimulation of the globus pallidus internus in patients with primary generalized dystonia: long-term results. J Neurosurg 2004; 101(2): 189-94.

[4] Coubes P, Echenne B, Roubertie A, et al. [Treatment of early-onset generalized dystonia by chronic bilateral stimulation of the internal globus pallidus. Apropos of a case]. Neurochirurgie 1999; 45(2): 139-44.

[5] Coubes P, Roubertie A, Vayssiere N, Hemm S, Echenne B. Treatment of DYT1-generalised dystonia by stimulation of the internal globus pallidus. Lancet 2000; 355(9222): 2220-1.

[6] Krauss JK, Loher TJ, Weigel R, Capelle HH, Weber S, Burgunder JM. Chronic stimulation of the globus pallidus internus for treatment of non-dYT1 generalized dystonia and choreoathetosis: 2year follow up. J Neurosurg 2003; 98(4): 785-92.

[7] Kupsch A, Benecke R, Muller J, et al. Pallidal deep-brain stimulation in primary generalized or segmental dystonia. N Engl J Med 2006; 355(19): 1978-90.

[8] Hodaie M, Wennberg RA, Dostrovsky JO, Lozano AM. Chronic anterior thalamus stimulation for intractable epilepsy. Epilepsia 2002; 43(6): 603-8.

[9] Wishart HA, Roberts DW, Roth RM, et al. Chronic deep brain stimulation for the treatment of tremor in multiple sclerosis: review and case reports. J Neurol Neurosurg Psychiatry 2003; 74(10): 1392-7.

[10] Mayberg HS, Lozano AM, Voon V, et al. Deep brain stimulation for treatment-resistant depression. Neuron 2005; 45(5): 651-60.

[11] Nuttin BJ, Gabriels L, van Kuyck K, Cosyns P. Electrical stimulation of the anterior limbs of the internal capsules in patients with severe obsessive-compulsive disorder: anecdotal reports. Neurosurg Clin N Am 2003; 14(2): 267-74.

[12] Visser-Vandewalle V. DBS in tourette syndrome: rationale, current status and future prospects. Acta Neurochir Suppl 2007; 97(Pt 2): 215-22.

[13] Coubes P, Vayssiere N, El Fertit H, et al. Deep brain stimulation for dystonia. Surgical technique. Stereotact Funct Neurosurg 2002; 78(3-4): 183-91.

[14] Starr PA, Vitek JL, DeLong M, Bakay RA. Magnetic resonance imaging-based stereotactic localization of the globus pallidus and subthalamic nucleus. Neurosurgery 1999; 44(2): 303-13; discussion 13-4.

[15] Vayssiere N, Hemm S, Zanca M, et al. Magnetic resonance imaging stereotactic target localization for deep brain stimulation in dystonic children. J Neurosurg 2000; 93(5): 784-90.

[16] Hariz MI. Complications of deep brain stimulation surgery. Mov Disord 2002; 17 (Suppl 3): S162-6. 
[17] Vayssiere N, Hemm S, Cif L, et al. Comparison of atlas- and magnetic resonance imaging-based stereotactic targeting of the globus pallidus internus in the performance of deep brain stimulation for treatment of dystonia. J Neurosurg 2002; 96(4): 673-9.

[18] Burke RE, Fahn S, Marsden CD, Bressman SB, Moskowitz C, Friedman J. Validity and reliability of a rating scale for the primary torsion dystonias. Neurology 1985; 35(1): 73-7.

[19] Baker KB, Tkach JA, Nyenhuis JA, et al. Evaluation of specific absorption rate as a dosimeter of MRI-related implant heating. J Magn Reson Imaging 2004; 20(2): 315-20.

[20] Brodkey JS, Miyazaki Y, Ervin FR, Mark VH. Reversible Heat Lesions with Radiofrequency Current. a Method of Stereotactic Localization. J Neurosurg 1964; 21: 49-53.

[21] Tronnier VM, Staubert A, Hahnel S, Sarem-Aslani A. Magnetic resonance imaging with implanted neurostimulators: an in vitro and in vivo study. Neurosurgery 1999; 44(1): 118-25; discussion 25-6.

[22] Georgi JC, Stippich C, Tronnier VM, Heiland S. Active deep brain stimulation during MRI: a feasibility study. Magn Reson Med 2004; 51(2): 380-8

[23] Baker KB, Tkach J, Hall JD, Nyenhuis JA, Shellock FG, Rezai AR. Reduction of magnetic resonance imaging-related heating in deep brain stimulation leads using a lead management device. Neurosurgery 2005; 57(4 Suppl): 392-7; discussion -7.

[24] Finelli DA, Rezai AR, Ruggieri PM, et al. MR imaging-related heating of deep brain stimulation electrodes: in vitro study. AJNR Am J Neuroradiol 2002; 23(10): 1795-802.

[25] Rezai AR, Finelli D, Nyenhuis JA, et al. Neurostimulation systems for deep brain stimulation: in vitro evaluation of magnetic resonance imaging-related heating at 1.5 tesla. J Magn Reson Imaging 2002; 15(3): 241-50.

[26] Rezai AR, Baker KB, Tkach JA, et al. Is magnetic resonance imaging safe for patients with neurostimulation systems used for deep brain stimulation? Neurosurgery 2005; 57(5): 1056-62; discussion 62.

[27] Spiegel J, Fuss G, Backens M, et al. Transient dystonia following magnetic resonance imaging in a patient with deep brain stimulation electrodes for the treatment of Parkinson disease. Case report. J Neurosurg 2003; 99(4): 772-4.

[28] Henderson JM, Tkach J, Phillips M, Baker K, Shellock FG, Rezai AR. Permanent neurological deficit related to magnetic resonance imaging in a patient with implanted deep brain stimulation electrodes for Parkinson's disease: case report. Neurosurgery 2005; 57(5): E1063; discussion E. 